\title{
PROGRESO CIENTÍFICO Y VERDAD
}

JUAN VÁZQUEZ

Universidad de Santiago de Compostela

En este trabajo se aborda el problema del desarrollo cientffico y su propósito es demostrar cómo a planteamientos aparentemente divergentes e históricamente enfrentados puede hacérseles converger en una misma dirección, en una dirección muy próxima al "realismo interno", propuesto por H. Putnam a partir de 1978 con la publicación de Meaning and the Moral Sciences, ${ }^{1}$ y que supone un planteamiento metateórico alternativo a los representados por el realismo y el instrumentalismo.

\section{Aproximación neopositivista}

La actual filosofía de la ciencia surge en el seno del neopositivismo como un estudio de la estructura de las teorfas científicas y sólo en una época bastante tardía, primero Kemeny y Oppenheim en 1956 (pp. 6-19) y luego Nagel en 1961 (cap. XI) se plantean por primera vez el problema de cómo explicar, en el marco de dicha concepción, ciertos procesos de cambio científico que parecían habituales o, al menos que ellos consideraron como los más significativos en la historia de la ciencia moderna.

1 De hecho H. Putnam formula por primera vez su argumento modeloteórico en 1976, en una comunicación ante la American Philosophical As8ociation. 
Hasta prácticamente finales del siglo XIX físicos y filósofos vieron cómo sectores muy diversos de la física eran absorbidos por la mecánica clásica. Y aunque a finales del XIX y comienzos del XX se tenía la impresión de que este imperialismo de la mecánica clásica había llegado a su fin, ese ideal de expansión de las teorías a dominios para los que en principio no habían sido planificadas o la absorción de unas teorías por otras más amplias se seguía viendo como una característica innegable y recurrente de la historia de la ciencia moderna. En la opinión de E. Nagel, había toda clase de razones para suponer que el tipo de desarrollo por absorción constituía el modelo más importante de evolución que seguiría experimentando la ciencia en el futuro (Nagel, 1961, p. 310). ${ }^{2}$

La expresión más habitual entre los neopositivistas para referirse al fenómeno de absorción es el de "reducción". Kemeny y Oppenheim titulan su artículo en 1956 "On Reduction” y el caṕtulo XI de La estructura de la ciencia de Nagel, que se convertira en representante de la tesis estándar del neopositivismo sobre la reducción, lleva por título "La reducción de teorías".

Aunque no todos los procesos reductivos son del mismo tipo, es interesante destacar que, en el marco de la concepción neopositiva de la ciencia, el término "reducción" se utiliza siempre como sinónimo de "deductibilidad". Tanto en las reducciones de tipo homogéneo como en las de tipo heterogéneo, si podemos hablar de reducción es porque se ha logrado establecer una relación de deducibilidad de la teoría reducida a partir de la teoría reductora, ya sea directamente, ya sea por medio de suposiciones adicionales que vinculan términos descriptivos específicos de la teoría que ha de reducirse con características expresables por los términos teóricos de la teoría reductora. Para utilizar sus propios ejemplos, la teoría galileana sobre la cáda de los cuerpos en la superficie de la tierra se reduce a

2 Siempre que en la bibliografía ce recoja la versión castellana de las obras, la traducción de los textos citedos se tomará de esa versión. 
la teoría newtoniana de la mecánica y la gravitación, porque las leyes de la primera son deducibles directamente de la segunda, al no haber diferencias cualitativas importantes entre los términos descriptivos de ambas teorías. En cambio la reducción de la termodinámica a la mecánica estadística o de las leyes de Kepler a la dinámica de Newton sólo son factibles mediante la introducción de suposiciones adicionales que pongan en conexión características expresadas por los términos teóricos de la teoría reductora con el significado de aquellos terminos descriptivos que son específicos de la teoría reducida.

Pero ya sea con la introducción de suposiciones adicionales o sin ella, el modelo de desarrollo científico implícito en el concepto de reducción, puesto que implica deducibilidad, responde a un modelo de desarrollo acumulativo en el que el contenido empírico representado por las viejas teorías probadas no se pierde ni se modifica, sino que éstas lo ceden a nuevas teorías más amplias; en estas últimas los nuevos logros se suman a los viejos, dando así lugar a los que más tarde va a ser designado como "concepción acumulativa del desarrollo científico", de la que los neopositivistas pasan por ser sus máximos representantes.

Las críticas de T. Kuhn a la concepción acumulativa del desarrollo científico así como la quiebra, parece que definitiva, del supuesto neopositivista de que los enunciados observacionales son teórica y científicamente neutrales, que sería el gran supuesto sobre el que en última instancia vendría a asentarse el concepto de reducción, parecen haber acabado con la posibilidad de seguir entendiendo el proceso del desarrollo científico como un proceso acumulativo de conocimientos. A los sumo, si algo queda de la vieja concepción es el ideal de convergencia: las teorías científicas convergen en el sentido de que las primeras con frecuencia son consideradas como casos límites de sus sucesoras. La base observacional de la teoría $t_{i-1}$ puede no ser la misma que la de $t_{i}$, pero apoyándose en el Principio de 
Caridad o Principio del Beneficio de la Duda (Putnam, 1975, pp. 272-290), suponemos que si los términos de $t_{i-1}$ tienen referentes entonces también los tendrán desde el punto de vista de $t_{i}$, siempre que $t_{i}$ contenga $t_{i-1}$ como un posible caso límite suyo.

\section{Plantemientos popperianos}

Frente al positivismo lógico Popper ha dedicado una atención especial al estudio del desarrollo cientifico. Se hace innecesario recordar que la definición de "verosimilitud", que él propuso como criterio de racionalidad con el cual poder evaluar el progreso cientifico, ha llegado a convertirse en uno de los problemas lógicos más importantes a los que se ha visto enfrentada la filosoffa de la ciencia en la década de los años setenta. ${ }^{3}$ Sin embargo, por muy importante que haya llegado a ser la noción de verosimilitud como medida del progreso científico, convendría distinguir entre el Popper de La lógica de la investigación cientifica, por una parte, y el Popper que pudiéramos denominar tarskiano o de la verosimilitud, por la otra.

Es cierto que tanto el Popper de La lógica de la investigación cientifica como el Popper tarskiano coinciden en postular que la ciencia no es un sistema de conocimientos seguros y bien establecidos. "Nuestra ciencia no es conocimiento (episteme): nunca puede pretender que ha alcanzado la verdad, ni siquiera el sustituto de ésta que es la probabilidad", se nos dice en último parágrafo de La lógica de la investigación cientlfica, y prosigue, "aunque no puede alcanzar ni la verdad ni la probabilidad, el esforzarse por el conocimiento y la búsqueda de la verdad siguen constituyendo los motivos más fuertes de la investigación cientifica" (Popper, 1962, p. 259).

3 En el artículo de Chris Bring: "Verosimilitude: Views and Reviews" se ofrece una presentación panorámica e información bibliográfica actualizada sobre el tema de la verosimilitud.

104 
Popper siempre ha mantenido que la búsqueda de la verdad constituye el ideal último al que se encamina el desarrollo del conocimiento científico; pero mientras que en La lógica de la investigación cientffica aborda el problema del desarrollo científico en el marco de su antiinductivismo falsacionista, sin comprometerse explícitamente con ningún concepto de verdad que no sea el concepto de verdad como coherencia, el Popper tarskiano o de la verosimilitud ya se ha comprometido con una determinada noción de verdad, con la noción de verdad como correspondencia y con la postulación de una verdad absoluta y total a la que debe encaminarse el conocimiento en su desarrollo.

No pretendo negar que no estuviese ya implícita la noción de verdad como correspondencia con los hechos en La lógica de la investigación científica, pero esa correspondencia no tiene por qué ser interpretada en el sentido de su realismo crítico posterior. Digamos que el Popper de La lógica de la investigación cientifica asume menos compromisos ontologicos y su realismo es perfectamente asimilable a lo que años más tarde $H$. Putnam va a calificar como "realismo interno". Como él mismo confesará en Conjeturas y refutaciones, "antes de conocer la teoría de la verdad de Tarski, me parecía más seguro y más económico discutir el criterio de progreso sin penetrar muy profundamente en el controvertido problema vinculado con el uso de la palabra 'verdad' " (Popper, 1965, p. 237). De hecho, como se acaba de indicar hace un momento, la única noción de verdad inherente a su antiinductivismo falsacionista es la noción de verdad como coherencia.

En una falsación empírica no son los hechos los que se toman en consideración, sino las relaciones de deducibilidad que median entre los enunciados teóricos de la ciencia, por una parte, y los "enunciados básicos", aceptados intersubjetivamente como válidos por la comunidad científica, por la otra. Como el propio Popper acaba confesando en el penúltimo parágrafo de La lógica de la investigación científica, 
en la lógica de la ciencia que he bosquejado es posible evitar el empleo de los conceptos de verdadero y falso: en su lugar pueden entrar consideraciones lógicas acerca de relaciones de deductibilidad. Así pues, no necesitamos decir, "la predicción $p$ es verdadera si la teoría $t$ y el enunciado $b$ son verdaderos"; en vez de ello, podemos decir que el enunciado $p$ se sigue de la conjunción (no contradictoria) de $t$ y $b$. Y cabe describir la falsación de una teoría de un modo semejante: no es menester que digamos que una teoría es falsa, sino solamente que la contradice cierto conjunto de enunciados básicos aceptados. (Popper, 1962, pp. 255-256)

Y como señala inmediatamente después, tampoco de los enunciados básicos tenemos por qué mantener que son 'verdaderos' o 'falsos', “ya que podemos interpretar su aceptación como el resultado de una decisión convencional y considerar los enunciados aceptados como resultado de tal decisión" (Popper, 1962, p. 256).

Sin llegar a pensar que K. Popper sea tan radical en su convencionalismo como ahora acaba de manifestársenos, lo cierto es que para él los enunciados básicos, por su proximidad a la experiencia, son más fiables que los téricos, o si se prefiere, es más fácil para la comunidad de científicos llegar a un acuerdo sobre su aceptabilidad o no aceptabilidad; con todo, no es menos cierto que Popper siempre se opuso a la existencia de enunciados últimos en la ciencia. Para decirlo de una manera sucinta y con sus propias palabras, su punto de vista es que "nuestro lenguaje habitual está lleno de teorías, que llevamos a cabo toda observación a la luz de teorías, que el prejuicio inductivista es lo único que lleva a muchos a creer que podría existir un lenguaje fenoménico, libre de teorías y distinguible de un 'lenguaje teórico'" (Popper, 1962, p. 57, nota 1).

No tiene, pues, sentido preguntarse por el valor de verdad de un enunciado, independientemente del sistema de creencias en el que es formulado y con relación al cual se hace significativa nuestra experiencia empírica, el ámbito de lo observable. En 
expresión de Putnam, "los 'objetos' no existen independientemente de los esquemas conceptuales" (Putnam, 1981, p. 61).

En resumen, y dando por supuesto que no es admisible racionalmente un conjunto de enunciados contradictorios, tendríamos que concluir que el único modelo de desarrollo científico que se sigue necesariamente de La logica de la investigación cientifica vendría determinado única y exclusivamente por la búsqueda ininterrumpida de un sistema de conjeturas o teorías que dé cuenta y, al mismo tiempo, sea consistente con el conjunto de enunciados básicos aceptados por la comunidad científica en cada momento de su historia.

Nos encontramos, pues, con un modelo de desarrollo científico no acumulativo, sino de eliminación de errores, cuya meta final, en el supuesto de que pudiera alcanzarse, no tendría por qué interpretarse como la búsqueda de la verdad, entendida en un sentido objetivo y absoluto, sino simplemente como la búsqueda de un sistema coherente de conjeturas teóricas explicativas y "enunciados básicos" aceptados. Un punto de vista, como luego tendremos ocasión de comprobar, muy próximo al de T. Kuhn y al "realismo interno" de H. Putnam y, por cierto, bastante alejado del "realismo crítico" del último Popper.

Tal vez por reacción contra la ola de relativismo que invade la filosofía de la ciencia en la década de los años sesenta, Popper evoluciona en su obra posterior hacia una actitud mucho más comprometida ontologicamente y que en absoluto venía exigida para su epistemología, si consideramos como lo más representativo de la misma su antiinductivismo falsacionista. En primer lugar, se compromete con la aceptación explícita del concepto de verdad como correspondencia, y ello, según Popper, porque "Tarski ha logrado establecer una teoría de la correspondencia de la verdad absoluta y objetiva, que se había vuelto sospechosa" (Popper, 1965, p. 273). En segundo lugar, con base en una combinación de las nociones de verdad y falsedad con la 
de contenido, propone la verosimilitud como nuevo criterio de progreso. ${ }^{4}$

Aunque no voy a entrar aquí en un análisis detallado del nuevo criterio de progreso científico formulado por Popper, es obvio que con su definición de verosimilitud, en términos de verdad y contenido, Popper ha querido ofrecernos un criterio con el cual evaluar teorías en función de su mayor o menor grado de aproximación a la verdad absoluta y objetiva; sin embargo no es menos cierto que pocos conceptos han originado tanta polémica en el seno de la filosofía de la ciencia como sus nociones cualitativa y cuantitativa de verosimilitud.

En primer lugar, el criterio de progreso científico basado en la noción de verosimilitud ya no sería aplicable a teorías supuestamente inconmensurables. En segundo lugar, como han demostrado Tìchy, Miller y Grünbaum, para cualesquiera dos teorías falsas diferentes $t_{1}$ y $t_{2}$ (y para Popper todas lo son potencialmente), partiendo de las definiciones popperianas de verosimilitud es posible demostrar que las teorías que tienen un elevado contenido de verdad también tienen un elevado contenido de falsedad o, lo que viene a ser lo mismo, que en contra de las definiciones de verosimilitud de Popper es posible demostrar que si la teoría $t_{1}$ tiene más contenido de verdad que $t_{2}$ también tiene más contenido de falsedad. En tercer lugar, como argumentará Newton-Smith, cualesquiera dos teorías mínimamente interesantes "contendrán el mismo número de verdades y el mismo número de falsedades, a saber, un número infinito" (Newton-Smith, 1981, p. 218), y no hay modo de comparar conjuntos infinitos de enunciados.

4 Cuando menos es discutible que el concepto de verdad que Tarski define para los lenguajes formales pueda aplicarse sin más a los lenguajes naturales y científicos, en los que no es fácil distinguir entre lenguaje y metalenguaje (requisito formal de la definición tarskiana de verdad). Por otra parte, no es menos problemático que la noción de verdad propuesta por Tarski pueda interpretarse como una definición de la verdad como correspondencia entre enunciados y hechos. 
Ya entre nosotros, el profesor José Sanmartín ha demostrado en el marco de la propuesta popperiana que "todas las teorías de un lenguaje dado $L$ tienen la misma medida de contenido de falsedad" (Sanmartín, 1984), lo que a su vez entraría en contradicción con la definición de verosimilitud que, en la formulación de Popper, exige que la verosimilitud de una teoría disminuya con el incremento de su contenido de falsedad. Por su parte el profesor Rivadulla, que ha hecho un repaso histórico bastante exhaustivo de todos los avatares por los que ha pasado la noción de verosimilitud, incluidas las sucesivas reformulaciones hechas por Popper, llega a la decepcionante conclusión de que los resultados alcanzados lo llevan "a un escepticismo respecto a la posibilidad de encontrar una definición de verosimilitud libre de objecciones" (Rivadulla, 1984, p. 173).

Como es también de sobra conocido, desde la primera reformulación de la noción de verosimilitud hecha por Tìchy en 1974 , se han producido no pocos intentos en la línea de proporcionar una definición correcta de verosimilitud, siendo los más conocidos los llevados a cabo por Niiniluoto $(1977,1980$, 1982 y 1987), que define la verosimilitud en términos de distancia entre constituyentes. Sin embargo, a diferencia de lo que acontecía con la definición de verosimilitud de Popper en la que se pretendía ofrecer un concepto de aproximación a la verdad absoluta y total, lo único que nos proporciona la definición de I. Niiniluoto es una aproximación a la verdad relativa $a$ un sistema conceptual o marco lingüístico dado. A su vez, el profesor Miguel A. Quintanilla en su artículo "La verosimilitud de las teorías" propone un nuevo criterio de verosimilitud, pero también relativo a la evidencia disponible, y no a la verdad total, como era el propósito de Popper.

En resumen, que el concepto de verosimilitud de Popper, como medida de aproximación a la verdad absoluta y total, se ha mostrado inconsistente y las nuevas formulaciones presentan como una de sus características más relevantes su relativización con respecto al marco conceptual proporcionado por el 
lenguaje de la teoría y a la evidencia disponible en cada momento histórico. Si cabe seguir hablando de una mayor aproximación a la verdad en el desarrollo del conocimiento, y yo creo que sí, esa verdad nada o muy poco tiene que ver con la verdad absoluta y total del último Popper; se parece más a la verdad postulada por el "realismo interno" de H. Putnam y de la que podemos considerar un anticipo la utilizada por Popper en La lógica de la investigación científica, a esa verdad que se genera con la coherencia de nuestras creencias entre sí y con nuestras experiencias, en cuanto experiencias representadas en nuestro sistema de creencias (Putnam, 1981, p. 59).

El positivismo lógico nos presentaba, en su versión estándar, el desarrollo científico como un proceso acumulativo de conocimientos en el que los viejos logros se sumaban a los nuevos. Ese proceso además de acumulativo era, evidentemente, convergente.

La revisión de Popper nos ha permitido observar, por una parte, el abandono de la concepción acumulativa y, por otra, la posibilidad de entender la nota de convergencia en dos sentidos totalmente distintos, según que nos refiramos al Popper de La lógica de la investigación cientifica o al Popper tarskiano o de la verosimilitud. Para el primero de ellos el desarrollo científico converge hacia teorfas cada vez más consistentes con los enunciados básicos aceptados como válidos por la comunidad científica. Para el Popper tarskiano esa convergencia se produce por la aproximación gradual del conocimiento a una supuesta verdad última, "absoluta" y "objetiva".

La revisión de Popper también nos ha servido para indicar al menos la mayor consistencia del Popper de La lógica de la investigación cientifica, y como los esfuerzos más recientes de Niiniluoto y algunos otros por reformular un concepto más adecuado de verosimilitud, implican un abandono de los compromisos ontológicos del Popper tarskiano. 


\section{Polémica Popper-Kuhn}

Lo que ahora me propongo demostrar es que el modelo de desarrollo científico propuesto por Popper en esa su primera obra fundamental, es compatible con el T. Kuhn y sólo recibirá de éste ligeras matizaciones, por lo demás perfectamente asumibles en el marco de una epistemología falsacionista. La fuerte polémica de enfrentamiento doctrinal que se ha originado entre popperianos y kuhnianos, a raíz de la publicación de la $E s-$ tructura de las revoluciones cientificas de Kuhn, sólo encuentra fundamento en los planteamientos tarskianos de Popper y en una incorrecta interpretación de la obra de Kuhn.

Entre las matizaciones más relevantes que Kuhn hace al modelo de desarrollo científico elaborado por Popper en La lógica de la investigación científica podría encontrarse su afirmación de que los científicos no siempre son tan críticos con sus teorías como Popper hubiera deseado. "Una vez que ha alcanzado el estatus de paradigma, una teoría científica se declara inválida sólo cuando se dispone de un candidato alternativo para que ocupe su lugar" (Kuhn, 1962, p. 128). Es decir, que no son los ejemplos en contra, los desajustes entre las teorías y algunos de sus enunciados básicos aceptados como válidos por la comunidad científica, los que motivan en exclusiva el abandono de una teoría. Para que ésta sea rechazada ha de disponerse además de un candidato alternativo mejor.

Popper, sin abandonar ninguno de los presupuestos básicos de su metodología, no tiene por qué no estar de acuerdo con esta matización. De hecho, cuando años más tarde propone la noción de verosimilitud como criterio de progreso, se da por supuesto - y así se recoge en las correspondientes definiciones de verosimilitud-que entre los componentes de las teorías en competencia se encuentran tanto enunciados verdaderos como enunciados falsos, luego éstos también subsisten en la teoría que se supone mejor. Lo único que las matizaciones de T. Kuhn ponen de manifiesto es la excesiva importancia que Popper atri- 
buyó a los ejemplos en contra, pero ello no significa que la falsación o demostración de inconsistencia entre las teorías y los enunciados básicos aceptados como válidos no jueguen un importante papel en el proceso del desarrollo científico, como también lo juegan las verificaciones o ejemplos a favor, siempre que éstos se entiendan no como validaciones o justificaciones definitivas del valor de verdad de las teorías sino como pruebas de su actual consistencia. Como reconocerá el propio T. Kuhn, las experiencias anómalas de Popper son importantes para la ciencia, ya que son la señal más clara de que algo no va bien y de que debe tratar de encontrarse una alternativa al paradigma existente (Kuhn, 1962, p. 228); pero lo que en modo alguno puede hacerse es interpretar las expresiones anómalas como si significaran falsación y rechazo, algo que el excesivo purismo de Popper sí había dejado entender y que tampoco es compatible con el "falsacionismo sofisticado" de I. Lakatos.

Hechas estas precisiones, convendría indicar que Kuhn, al igual que Popper, es un fiel defensor del desarrollo cientifico y cree que este desarrollo "es, como la evolución biológica, unidireccional e irreversible" (Kuhn, 1970, p. 432). En este sentido carecen de fundamento las acusaciones de relativismo de que ha sido objeto. Tampoco niega T. Kuhn que no haya buenas razones para elegir entre dos o más teorías. Si recordamos el conjunto de esas buenas razones ofrecidas por K. Popper en "La verdad, la racionalidad y el desarrollo del conocimiento científico", con base en las cuales una teoría $t_{2}$ es preferible a $t_{1}$, y comparamos esas buenas razones con las que nos ofrece T. Kuhn en el parágrafo 5 de "Consideración en torno a mis críticos" o también en el parágrafo 5 de la "Posdata: 1969" a la Estructura de las revoluciones cientificas, constatamos que esas razones son prácticamente las mismas: "exactitud, alcance, simplicidad, posibilidad de dar frutos, etc."; (Kuhn, 1970, p. 429; 1969, p. 304). Es más, T. Kuhn afirma expresamente que las buenas razones que él reconoce que existen en el debate entre paradigmas no son diferentes de las 
comúnmente registradas por los filósofos de la ciencia. Lo único que mantiene T. Kuhn es que esas razones no son compulsivas. Y aunque es de vital importancia para el desarrollo de la ciencia que se enseñe a los científicos a valorar esas características y que se ofrezcan ejemplos que las ilustren en la práctica, ya que "si no se aferrasen a valores como estos, sus disciplinas se desarrollarían de manera muy diferente" (Kuhn, 1970, p. 429); no obstante, T. Kuhn insiste en que "tales razones más constituyen valores para utilizar al hacer elecciones que reglas de elección" (Kuhn, 1970, p. 430).

Los científicos que comparten ese mismo sistema de buenas razones para elegir entre teorías en competencia pueden, sin embargo, hacer elecciones distintas en la misma situación concreta y no por ello cabe acusarlos de irracionales. Los motivos son obvios: difícilmente por no decir nunca, se da el caso de que dos teorías compartan los mismos valores y en el mismo grado. Una teoría es más simple, pero la otra es más exacta; la que en estos momentos ha sido más probada presenta, sin embargo, menos posibilidades de dar nuevos frutos en el futuro, etc., etc. Y no está claro que en todas las circunstancias deban anteponerse unos valores a otros. En unos casos será preferible la simplicidad a la exactitud, porque lo que nos interesa es tan sólo un resultado aproximado, pero en otros, en los que la exactitud de los resultados afecte a la validez del experimento, será más importante la exactitud que la simplicidad. Y ¿́por qué no va a ser mejor para los propios investigadores e incluso para el desarrollo de la ciencia que algunos de los jóvenes científicos se embarquen en la investigación de teorías poco probadas pero que, en principio, prometen abrirles nuevos ámbitos de la experiencia, que seguir trabajando en el desarrollo de una pequeña parcela de teorías ya establecidas, cuando saben de antemano que el resultado de su trabajo tendrá un escaso valor?

Creo que T. Kuhn está en lo cierto y ésta es una matización que K. Popper debe aceptar, que "simplicidad, alcance, posibilidad de dar frutos, e incluso exactitud pueden ser juzgados 
de manera muy diferente (lo que no es lo mismo que decir que puedan ser juzgados arbitrariamente) por personas diferentes" (Kuhn, 1970, p. 430).

La elección de una teoría o de un programa de investigación, como dice I. Lakatos, lleva consigo riesgos importantes, especialmente en sus primeras etapas. Pero si todos los miembros de la comunidad científica aplicasen los valores del mismo modo arriesgado y no corriese cada uno su riesgo particular, esos valores que hay que tener en cuenta, más que una guía para la investigación, se convertirían en una rémora. La cantidad de contenido verdadero o falso de una teoría, al margen de los problemas que presenta el averiguarlo, no es más que uno de los criterios que el científico puede seguir en la elección de teorías.

Nuevamente tendría que insistir en que estas matizaciones hechas por T. Kuhn no implican una invalidación del importante papel que K. Popper hizo jugar a la comprobación empírica en el proceso del desarrollo científico. No hay ningún incoveniente por parte de T. Kuhn en admitir que una de las características en que las nuevas teorías suelen aventajar a sus predecedoras es en su concordancia con la base experimental. El problema o la disparidad radical entre los puntos de vista de T. Kuhn y K. Popper está en cómo se entienda esa concordancia. Si nos referimos al Popper de La logica de la investigación científica esa concordancia no es más que una relación de coherencia entre conjeturas teóricas y enunciados básicos. Y aunque K. Popper no quiso por aquel entonces entrar en el análisis de la fundamentación empírica de los enunciados básicos, no sólo no veo ningún inconveniente, sino que me parece lo más acertado y consistente con la epistemología no comprometida ontológicamente del primer Popper, interpretar los enunciados básicos como descripciones de nuestras experiencias, en cuanto experiencias representadas en nuestro sistema de creencias. Para ser más explícito, fuera cual fuera la convicción interna de K. Popper, no hay nada en la epistemología de $L a$ 
lógica de la investigación científica que nos fuerce a interpretar las conjeturas téricas y los enunciados básicos de la ciencia como representaciones "de lo que está realmente ahî", de lo que él va a designar en su epistemología posterior como "mundo uno", y no, como cree T. Kuhn, de "lo que la naturaleza realmente semeja", es decir, de aquello como lo que la naturaleza es experimentada en el marco conceptual proporcionado por las teorías.

El que acepta la primera de las interpretaciones se compromete con la existencia de un mundo en si, que consta de alguna totalidad fija de objetos independientes de la mente, y se ve forzado a entender el desarrollo científico como un proceso de acercamiento a una supuesta verdad "objetiva", "absoluta" y "total". Muchos filósofos de la ciencia, entre ellos el Popper tarskiano o de la verosimilitud, se han comprometido con esta interpretación; y, como nos dirá T. Kuhn, "lo que ellos desean es comparar teorías como representaciones de la naturaleza, como enunciados acerca de 'lo que está realmente ahí'. Aun dando por supuesto que dadas dos teorías que han existido históricamente, ninguna es verdadera, ellos buscan, no obstante, un sentido en el que la última sea una mejor aproximación a la verdad" (Kuhn,1970, p. 433).

T. Kuhn, sin embargo, no cree que haya fundamento alguno para embarcarse en esa interpretación del desarrollo científico. Lo único que puede constatarse históricamente es que, por ejemplo, la mecánica de Newton mejora a la aristotélica y que la de Einstein perfecciona la de Newton, como instrumento para resolver enigmas (Kuhn, 1969, p. 314). 0, lo que vendría a ser lo mismo, que, vistas a posteriori, cada una de esas teorías se nos revela más adecuada que sus predecesoras porque es más consistente con la base experimental o conjunto de enunciados básicos aceptados como válidos por la comunidad científica. Pero lo que en absoluto puede hacerse es inferir, a partir de esa mejora en las consecuencias empíricas de las teorías, la noción de un paralelo entre la ontología de esas mismas teorías y su 
contraparte "real" en la naturaleza. Máxime, insistira Kuhn, cuando la comparación de teorías en la historia no da lugar a pensar que sus ontologías se aproximen hacia límite alguno (Kuhn, 1970, p. 434). Además, ese salto dado por Popper del plano experimental al paralelismo ontologico supone una violación del teorema que dice que cualquier conjunto finito de consecuencias de una teoría dada puede ser derivado también de otra teoría incompatible con ella.

Un segundo argumento utilizado también por T. Kuhn en contra del modelo de desarrollo científico que se sigue de interpretar la noción de concordancia como correspondencia con "lo que está realmente ahr" tiene que ver con las nociones kuhnianas de "revolución científica" e "inconmensurabilidad". Según Kuhn, la definición de verosimilitud o grado de aproximación a la verdad "absoluta" y "objetiva" propuesta por Popper supone que "quienes proponen teorías en competencia comparten un lenguaje neutral adecuado para la comparación de los informes de observación" (Kuhn, 1970, p. 434) y T. Kuhn no cree que exista un tal lenguaje neutral de observación, sino que como él mismo considera haber puesto de manifiesto a lo largo de la Estructura de las revoluciones cientificas, con los cambios de paradigma no sólo se producen modificaciones en la ontología de las teorías sino también en la estructuración de la base observacional, de tal modo que los miembros de comunidades que comparten teorías en competencia con frecuencia tienen también percepciones distintas. La base observacional no es, pues, independiente del sistema de creencias de los sujetos, lo que no significa que uno pueda percibir lo que quiera o que nuestras percepciones no tengan un fundamento empírico:

Decir que los miembros de grupos diferentes pueden tener distintas percepciones cuando se enfrentan a un mismo estímulo, no implica precisamente que ellos no puedan tener ninguna percepción en absoluto. En muchos ambientes un grupo que no supiera distinguir los lobos de los perros no podría perdurar; ni hoy podría un grupo de físicos nucleares sobrevivir como científicos 
si fueran incapaces de reconocer las huellas de las partículas alfa y de los electrones; precisamente debido a que tan escasas maneras de ver lo logran, es que aquellas que han resistido a los usos de las pruebas de grupo, son dignes de transmitirse de generación en generación. (Kuhn, 1969, p. 299)

En definitiva, que mientras el Popper tarskiano, a partir del mayor grado de consistencia de las últimas teorfas con los enunciados básicos aceptados, da el salto hacia la postulación de una verdad "absoluta" y "objetiva" de correspondencia con los hechos, T. Kuhn se niega a dar ese paso, por considerarlo injustificado. Frente a la verdad "objetiva" y "absoluta" del Popper tarskiano, Kuhn sólo acepta una noción intrateórica de verdad, perfectamente asimilable al uso explícito que hizo de esa noción Popper en La lógica de la investigación cientéfica. “Los miembros de una comunidad científica dada estarán generalmente de acuerdo en cuales son las consecuencias de una teoría común que pasan la prueba de la experimentación y son por lo tanto verdaderas, cuáles son falsas a medida que se aplica la teoría, y cuáles están todavía sin someter a contraste" (Kuhn, 1970, p. 433). En ese contexto, el uso intratérico del término "verdad" no le parece problemático a T. Kuhn. Al fin y al cabo, no se trata más que de comparar consecuencias empíricas de una teorfa con los resultados de la experimentación que se llevan a cabo en el marco de esa misma teorfa. Pero mientras no se demuestre la existencia de un "lenguaje neutral de observación", concluye Kuhn, no estamos autorizados a utilizar el término "verdad" como si el paso de contextos intrateóricos a contextos interteóricos careciese de importancia.

En resumen, que para T. Kuhn si resta algún uso legítimo del término "verdad" es el de coherencia de nuestras creencias entre sí y con nuestras experiencias, en cuanto experiencias representadas en nuestro sistema de creencias.

Puesto que el primero de los argumentos utilizados por T. Kuhn en contra de la opinión del último Popper de considerar a las teorías científicas como proyectos de aproximación 
a una supuesta descripción última de "lo que está realmente ahî" se basa, en una de sus partes, en la subdeterminación de las teorías a partir de la base experimental, y puesto que ese mismo argumento, el de la subdeterminación, es el que con más frecuencia han utilizado los instrumentalistas en contra del realismo científico, quisiera hacer una o dos matizaciones al respecto.

En primer lugar, y al margen de cuál pueda ser en este sentido el punto de vista de T. Kuhn, el paso que dieron algunos instrumentalistas como Poincaré o Duhem para concluir, con base en el teorema de la subdeterminación, que los términos teóricos de la ciencia no se refieren a nada en el mundo real sino que equivalen a reglas cuya única misión es la de facilitar la extracción de consecuencias empíricas, a la vez que posibilitan una labor de síntesis y economía intelectual, está tan poco justificado como el que ha dado K. Popper en el sentido contrario.

Si hay una subdeterminación de las teorías a partir de los datos, lo único que se puede concluir no es que los términos teóricos de la ciencia no se refieran a, sino que su referencia es siempre conjetural. Por lo demás, y como ha replicado ya más de un realista, no tiene ningún sentido conceder valor descriptivo a los enunciados empíricos de la ciencia y negárselo a los teóricos, a menos que estemos dispuestos a establecer un corte radical entre el nivel teórico por un lado y el observacional por el otro, algo en lo que ya nadie parece creer a raíz de las críticas de Quine, Hanson, Kuhn, Sellars, Shapere y tantos otros.

En segundo lugar, y esto es lo que voy a tratar de demostrar en las páginas que siguen, si tomamos como punto de partida las tesis en común y menos controvertidas de Khun y Popper, la ruta a seguir en la investigación futura debe alejarse por igual de las que hasta ahora se han venido señalando desde los planteamientos realistas e instrumentalistas, en cuanto unos y otros se hayan comprometido con la existencia de cosas en sí, independientes de la mente. Esta ruta apunta, creo yo, a una 
nueva dirección tanto en el análisis y explicación del desarrollo científico como en el no menos problemático concepto de verdad. Una dirección muy próxima a la representada por el realismo interno de $\mathrm{H}$. Putnam.

\section{El desarrollo científico en la línea del realismo interno}

Puede parecer parad6jico que haya comenzado por indicar que la nueva dirección en la que deben encaminarse las investigaciones epistemologicas sobre el desarrollo científico se alejan por igual tanto de los planteamientos realistas como de los instrumentalistas y, luego, haga coincidir esa nueva dirección con la diseñada por $H$. Putnam en el marco de su realismo interno. Alguien podría argumentar que, si Putnam califica a su nuevo planteamiento de "realismo interno", contraponiéndolo al "realismo metafísico" o "externalista", es porque, por muy alejado que éste esté del realismo metafísico, su punto de vista se sitúa en una línea más próxima al realismo que al instrumentalismo. Sin embargo, eso no es así, lo que tampoco quiere decir que debamos aproximarlo a los planteamientos instrumentalistas.

Tal vez resulte confusa la utilización del término "realismo" por parte de Putnam, en cuanto denotativo de su propia actitud, por más que luego se le matice con el calificativo de "interno". A este respecto quisiera comenzar por indicar que Ralph C. S. Walker, en su reciente obra The Coherence Theory of Truth, califica la teoría de la verdad de Putnam de coherentista. Por su parte ya Raimo Tuomela, en su ensayo de 1979 "Putnam's Realism", era de la opinión de que la clase de realismo interno que Putnam empezaba por aquel entonces a defender podría muy bien ser clasificado de "coherentismo". Y Gerald Vision, en Modern Anti-Realism and Manufactured Truth, considera a su vez a Putnam como un representante del antirrealismo. Personalmente no creo que pueda tacharse al "realismo interno" de Putnam de coherentista y menos todavía aun de antirrea- 
lista, a no ser que se tome como telón de fondo el realismo metaftsico o externalista, contra el que Putnam ha venido dirigiendo sus críticas desde 1976. Tanto en The Many Faces of Realism (Putnam, 1987, p. 17), como en Representation and Reality (Putnam, 1988, p. 114), el propio Putnam se muestra insatisfecho con la expresión "realismo interno" y confiesa que tal vez hubiera sido más acertado calificar a su nueva actitud de "realismo pragmático". No importa mucho que la denominación sea una u otra; tanto el término "interno" como el término "pragmático" reflejan aspectos importantes de la nueva perspectiva en la que se sitúan los planteamientos de Putnam.

A modo de comparación y en un primer proyecto de aproximación a esa nueva perspectiva representada por el realismo interno, cabría indicar que mientras Popper, en su huida del fantasma del relativismo, evoluciona desde una epistemología no comprometida ontológicamente hacia un realismo muy próximo al realismo metafísico —en lo que éste tiene de compromisos ontológicos-, la evolución de Putnam se produce en el sentido contrario; desde una primera actitud comprometida con el realismo metafísico deriva cada vez más hacia una epistemología libre de compromisos ontológicos. En esa misma línea de comparación cabría señalar además que discrepancias similares a las que hemos observado entre T. Kuhn y el Popper tarskiano, en relación con el concepto de verdad absoluta y objetiva y del desarrollo cientifico como un proyecto de aproximación a la verdad total, son las que ahora vamos a detectar en el enfrentamiento de Putnam con el realismo metafísico.

Según la caracterización que el propio Putnam hace del realismo metafísico, éste vendría definido por la conjunción de las tres tesis siguientes:

1. Existe un mundo teórico-independiente. 0 , como se nos dice literalmente en Razón, verdad e historia, "el mundo consta 
de una totalidad fija de objetos independientes de la mente" (Putnam, 1981, p. 49).

2. "Hay exactamente una descripción verdadera y completa de 'como es el mundo" (Putnam, 1981, p. 59). Es decir, que en el supuesto de que dos teorías fueran descripciones completas y verdaderas del mundo, las diferencias entre ellas serían meramente notacionales (Putnam, 1983, p. 211).

3. "La verdad supone una especie de relación de correspondencia entre palabras o signos mentales y cosas o conjuntos de cosas externas" (Putnam, 1981, p. 59). Lo que equivale a postular que la verdad es radicalmente no epistémica.

Antes de pasar a presentar, también de una manera sucinta y en tesis correlativas, el punto de vista representado por el realismo interno, quisiera llamar la atención sobre un hecho que considero importante en relación con el tema que nos ocupa. El realismo metafísico, en la versión que Putnam nos acaba de ofrecer de él, es perfectamente asumible por el Popper tarskiano, choca frontalmente con los planteamientos de Kuhn y podría pero no tendría por qué ser asumible por el Popper de La lógica de la investigación científica.

La opinión de Putnam al respecto es sencilla y llanamente que el realismo metafísico representa una metateoría incoherente, incapaz de explicar, sin incurrir en inconsistencias, la relación que media entre cualquier teoría correcta y la totalidad o una parte del mundo.

Por su parte, el realismo interno no es que niegue todas y cada una de las tesis propuestas por el realismo metafísico, como afirma Raimo Tuomela en Science, Action, and Reality (Putnam, 1985, p. 96). Si lo hiciera así estaría incurriendo en las mismas o similares incoherencias en las que, según Putnam, incurre el realismo metafísico. Lo que se hace en el realismo interno es adoptar una nueva perspectiva, diseñar un marco metateórico distinto desde el que deben ser analizadas las nociones de verdad, desarrollo científico y, en general, el conjunto 
de correlaciones que se supone que deben mediar entre cualquier teoría y el mundo.

En paralelo con la sucinta caracterización que antes se ha hecho del realismo metafísico, también el realismo interno podría quedar sintetizado en la conjunción correlativa de los tres postulados siguientes:

1'. "¿De qué objetos consta el mundo? es una cuestión que sólo tiene sentido preguntar dentro de una teoría o descripción" (Putnam, 1981, p. 49).

$2^{\prime}$. "Muchos filósofos internalistas, aunque no todos, sostienen además que hay más de una teoría o descripción del mundo 'verdadera'" (Putnam, 1981, p. 49).

3 '. "La 'verdad' es una especie de aceptabilidad racional (idealizada) - una especie de coherencia ideal de nuestras creencias entre sí y con nuestras experiencias, tal como estas experiencias son ellas mismas representadas en nuestro sistema de creencias - y no una correspondencia con 'estados de cosas' independientes de la mente o del discurso" (Putnam, 1981, pp. 49-50).

Si como anteriormente se ha hecho con relación al realimo metafísico, comparamos ahora los puntos de vista de Popper y Kuhn con el realismo interno, tendríamos que decir que el realismo interno coincide casi literalmente con los planteamientos de Kuhn, choca frontalmente con el Popper tarskiano y podría ser subscrito, sin incurrir en contradicción, por el Popper de La lógica de la investigación cientffica.

Obsérvese, como se ha indicado ya hace un momento, que los tres postulados en los que, siguiendo a Putnam, se ha tratado de sintetizar el realismo interno no suponen una negación del realismo metafísico sino un cambio de pespectiva. Lo que habría que decir desde el postulado $\mathbf{l}^{\prime}$ del realismo interno no es que la tesis 1 del realismo metafísico sea falsa sino que carece de sentido, que tan vacuo es afirmar la existencia de un 
mundo teórico independiente como negarla. Si a lo único que podemos tener acceso, como se mantiene en el postulado $1^{\prime}$ de realismo interno, es a un mundo de cosas conceptualizadas, hablar de un mundo libre de toda conceptualización - ya sea que esto se haga para afirmar su existencia o para negarlacarece de sentido.

De una manera consecuente, tampoco en el postulado 3 estaremos autorizados a establecer la verdad como una especie de relación de correspondencia entre palabras o signos mentales y cosas o conjuntos de cosas externas, si por cosas externas entendemos, como se supone que está entendiendo el realista metafísico, cosas no conceptualizadas. Si alguien postula que el mundo está constituido por alguna totalidad fija de "cosas en sr', independientes de la mente, entonces ya nunca podrá saber si el mundo tal como se le presenta se corresponde o no con lo que esas cosas son en $s i$, porque para poder saberlo tendría que tener acceso, por una parte, a las "cosas en s $f$ ", con independencia de toda conceptualización, y por otra, a las cosas ya conceptualizadas, pero sólo la segunda de las posibilidades es viable, puesto que no somos el Ojo Divino y en el preciso momento en el que un sujeto cualquiera accediese a esa supuesta realidad independiente de la mente, esa realidad ya no sería en sí, sino una realidad conceptualizada. En resumen, y esto es lo que dice el realismo interno, que "nosotros no sabemos acerca de lo que estamos hablando cuando hablamos acerca de las "cosas en sf'" (Putnam, 1987, p. 36).

La única correspondencia de la que realmente podemos hablar con sentido es la que se establece, como ya nos decía Kuhn y ahora repite Putnam, entre las palabras o signos mentales y las cosas o conjuntos de cosas conceptualizadas (Putnam, 1981 , p. 64). Para decirlo con sus propias palabras: "si, como mantengo, los propios objetos son tanto construidos como descubiertos, son tanto producto de nuestra invención como del factor 'objetivo' de la experiencia, el factor independiente de nuestra voluntad, entonces los objetos pertenecen intrínseca- 
mente a ciertas etiquetas; porque esas etiquetas son las herramientas que usamos para construir una versión del mundo en la que tales objetos ocupan un lugar preeminente" (Putnam, 1981 , p. 63). Que haya un único sistema de conceptualización válido o que éste sea plural no es algo que pueda establecerse tampoco de una manera $a$ priori ni desde el realismo metafísico ni desde el realismo interno. Si el sistema de conceptualización fuese único no habría más que una teoría o descripción "verdadera" del mundo, pero si el sistema de conceptualización es plural podrá haber más de una teoría o descripción igualmente "verdadera", pero como ninguna de estas dos posibilidades puede ser decidida al margen de la investigación empírica del sistema o los sistemas de conceptualización vigentes, una y otra actitud son igualmente compatibles con el realismo interno, tesis $2^{\prime}$.

Una vez postulada la existencia de un mundo de entidades independientes de la mente y del discurso, entonces ya nada podemos decir con sentido de él. Esta idea fundamental es la que, en última instancia, hace incoherentes a la mayoría de los realismos y da sentido a las críticas de Putnam en contra del realismo metafísico.

Si se postula la existencia de un mundo teórico-independiente (tesis 1) y se postula también que lo que hace verdadera a una sentencia cualquiera de un lenguaje $L$ es la correspondencia entre los términos de $L$ y las entidades del mundo (tesis 3 ), entonces, puesto que se trata de un mundo independiente de la mente, nada puede objetarse a quién, como Putnam, mantiene que bajo esos presupuestos, es posible demostrar la existencia de más de una relación de referencia privilegiada. A tal efecto, Putnam formula el siguiente teorema:

Sea $L$ un lenguaje con los predicados $F_{1}, F_{2}, \ldots, F_{k}$ (no necesariamente monádicos). Sea $I$ una interpretación que asigna una intensión a cada predicado de $L$. Entonces, si $I$ es no trivial, en el sentido de que al menos un predicado tiene una extensión que ni es vacía ni universal al menos en un mundo posible, existe 
una segunda interpretación $J$ que no coincide con $I$, pero que satisface las mismas oraciones que $I$ en cada mundo posible. (Putnam, 1981, p. 215)

En la demostración de este teorema Putnam trata las intensiones de los predicados como funciones de mundos posibles a conjuntos de objetos en esos mundos posibles, y la estrategia a seguir en la demostración se basa en la elección de un predicado cualquiera $F_{i}$ de $L$ y asignarle una interpretación $J$ distinta de $I$, de tal modo que la extensión de $F_{i}$ para la interpretación $J$ en cada mundo posible venga determinada por una función que selecciona en cada uno de esos mundos una permutación de la extensión asignada a $\boldsymbol{F}_{\boldsymbol{i}}$ por la interpretación I (Putnam, 1981, pp. 215-216).

Evidentemente, el modelo asf construido a partir de la interpretación $J$ es en todo isomorfo con el determinado por la interpretación $I$, y, en virtud del teorema del isomorfismo, en cualquier mundo posible en el que una oración resulte verdadera para la interpretación I también lo será para la interpretación $J$, a pesar de que sus referentes últimos varíen de una a otra interpretación.

Aunque en su aplicación a los lenguajes formales de primer orden, nadie pone en duda la corrección formal del Teorema de Löwenheim-Skolem, la extensión que Putnam hace de él a los lenguajes naturales, incluidos los cientificos, si que ha sido duramente criticada por Koethe (1979), Merrill (1980), Pearce y Rantala (1982), Lewis (1984), Tuomela (1979 y 1985), etc.

Sin embargo, y siempre desde la aceptación previa de las tesis 1 a 3 del realismo metafísico, puesto que en el marco de esa perspectiva externalista "la verdad de una teoría no consiste en su ajuste con el mundo conforme éste se presenta al

5 Putnam desarrolla esta misma tesis (su argumento modelo-teoretico) en: Meaning and the Moral Sciences, "Models and Reality" The Journal of Symbolic Logic, no. 45, 1980, Realism and Reason. Philosophical Papers (vol.3), "Models Theory and the 'Factuality' of Semantics" (en Alexander George (ed.), Reflections on Chomsky, Basil Blackwell, Cambridge, 1989). 
observador u observadores (la 'verdad' no es relacional en este sentido), sino en su correspondencia con el mundo tal como es en sí mismo" (Putnam, 1981, p. 60), mientras se respeten todas las constricciones teórico-prácticas exigidas por el uso (consistencia, completitud, simplicidad, capacidad predictiva, etc.), nada hay en la experiencia empírica que nos fuerce a pensar en la existencia de una única referencia privilegiada para los términos del lenguaje $L$.

Contraargumentar con base en una semántica sellarsiana, como lo hace R. Tuomela en Science, Action, and Reality, que la referencia en los lenguajes naturales es una noción intralingüística que viene determinada por una pluralidad de factores psico-socio-históricos, o que, en cualquier caso, en circunstancias normales los sujetos humanos tienen éxito en el acto de referencia (Tuomela, 1985, p. 99-100), no constituye en realidad, ninguna réplica a los argumentos de Putnam en contra del realismo metafísico.

Putnam no niega que los sujetos humanos no tengamos éxito en nuestros actos de referencia, ni que los términos de nuestro lenguaje carezcan de referentes reales en el mundo, "conforme éste se presenta al observador u observadores", lo único que Putnam trata de demostrar es la propia incoherencia interna de la perspectiva filosófica representada por el realismo metafísico. Me atrevería a decir que tanto el argumento modeloteórico, al que acabamos de hacer referencia, como los argumentos de tipo semántico, se encaminan más que a defender una pluralidad de relaciones de correspondencia igualmente válidas, a demostrar sencilla y llanamente que el realismo metafísico, con su postulación de "un mundo de entidades independientes de la mente y del discurso", se ha autoincapacitado para poder legitimar ningún tipo de relación de "correspondencia" entre palabras o signos mentales y cosas o conjuntos de cosas "externas".

Una vez que se ha postulado la existencia de un mundo independiente de la mente, como la única relación de correspon- 
dencia a la que podemos tener acceso es a la que se establece entre las palabras o signos mentales y el mundo "conforme éste se presenta al observador u observadores", entonces, con relación al mundo tal como es en sí mismo, nada puede objetarse a la demostración de Putnam de que, bajo ese supuesto, es posible construir muchas relaciones de correspondencia diferentes, pero todas ellas igual mente legítimas. "Porque, suponiendo un mundo de entidades independientes de la mente y del discurso (y esto es lo que presupone el punto de vista que estamos discutiendo) hay, como hemos visto, muchas 'correspondencias' diferentes que representan relaciones de referencia posibles $o$ candidatas (infinitamente muchas, de hecho, si hay una cantidad infinita de cosas en el universo)" (Putnam, 1981, p. 58).

Una vez que se ha postulado la existencia de un mundo independiente de la mente y del discurso, entonces es relativamente fácil demostrar con base en ese supuesto, como lo hace Putnam, que si somos cerebros en un cubeta no podemos decir o pensar que lo somos y que el mundo nocional de los cerebros en una cubeta se halla incapacitado para referirse a objetos "externos" y fijar la referencia. ${ }^{6}$ En definitiva, que la tesis 1 del realismo metafísico convierte a las tesis 2 y 3 en afirmaciones gratuitas. Por el contrario, para un internalista como Putnam la situacion es completamente distinta:

6 Para demostrar que el estado psicológico o mundo nocional del hablante no determina la extensión Putnam se sirve del análisis de tres experimentos mentales:

a) el caso de "agua" en la Tierra y en Tierra Gemela en 1750,

b) el caso de "aluminio" y "molibdeno" y

c) el caso de "olmo" y "haya".

Con el análisis de estos tres ejemplos Putnam trata de mostrar cómo dos hablantes pueden estar en el mismo estado psicológico y, a la vez, diferir la extensión de un mismo término del idiolecto del primero al idiolecto del segundo. Para Putnam la extensión es en parte determinada socialmente (división del trabajo linguíatico) y en parte indexicalmente (Putnam, 1975, p. 245). 
desde una perspectiva internalista, los signos tampoco corresponden intrínsecamente a objetos con independencia de quién o cómo los emplee. Pero un signo empleado de un modo determinado por una determinada comunidad de usuarios puede corresponder a determinados objetos dentro del esquema conceptual de esos usuarios. Los “objetos" no existen independientemente de los esquemas conceptuales. Desmenuzamos el mundo en objetos cuando introducimos uno u otro esquema descriptivo, y puesto que tanto los objetos como los símbolos son internos en el esquema descriptivo, es posible indicar cómo se emparejan. (Putnam, 1981, p. 61)

Ahora bien, si como postula R. Tuomela en el capítulo 6 de Science, Action, and Reality, es fundamental para todas las versiones del realismo mantener que "existen particulares reales (objetos, eventos, procesos, etc.) que son independientes de la mente" (Tuomela, 1985, pp. 106-107), entonces yo tendría que decir, si he entendido verdaderamente a Putnam, que su "realismo interno" nada tiene que ver con los realismos que aceptan como uno de sus postulados la existencia de "cosas en sî" independientes de la mente. Como se nos dice expresamente en The Many Faces of Realism, "la adopción del realismo interno es la renuncia a la noción de la 'cosa en sî" (Putnam, 1987, p. 36); y no porque nosotros no podemos conocer las cosas en sí mismas, eso también se mantiene, entre otros muchos, por parte del "realismo referencial" de Rom Harré (1985) y del "realismo causal interno" de R. Tuomela (1985). La renuncia a la noción de la “cosa en sî" en el realismo interno obedece al autoconvencimiento de Putnam de que esa noción es en sí misma autocontradictoria.

Quienes aceptan la noción de la "cosa en s $\Gamma$ " incurren necesariamente en inconsistencias, tanto si luego afirman su cognoscibilidad, como puede ser el caso del realismo metafísico, como su incognoscibilidad, como mantienen Tuomela, Rom Harré, R. Bhaskar y el propio K. Popper. 
Negar sentido a la noción de "lo en sî" no significa que no se pueda seguir hablando de objetos, eventos y proceso externos, nadie lo pone en duda, lo que no tiene sentido alguno es afirmar que esos objetos, eventos y procesos existen con independencia de toda elección conceptual, como pretenden la mayoría de los realistas.

Cuando R. Tuomela, en su caracterización del "realismo causal interno", enuncia como primer axioma fundamental que "existen particulares reales (objetos, eventos, procesos, etc.) que son independientes de la mente" o cuando el realismo metafísico, en la versión que el propio Putnam nos ofrece de él, postula que "el mundo consta de alguna totalidad fija de objetos independientes de la mente", lo que habría que indicarles desde el realismo interno no es que esos enunciados sean falsos. Si lo hiciéramos así estaríamos incurriendo en un error similar al que cometen quienes los postulan. Tan incorrecto y falto de sentido es afirmar la existencia de "cosas en sî" como el negarla. Lo único que acontece con los enunciados acerca de lo en sí es sencillamente que carecen de sentido, que quienes los formulan, como nos decía Putnam, no saben de lo que están hablando.

Frente al realismo metafísico o frente a cualquier tipo de realismo que se haya comprometido con la existencia de estados de cosas independientes de la mente, el realismo interno mantiene que lo que sea el mundo, independientemente de cómo es experimentado a través de nuestros marcos conceptuales, no es algo que pueda ser dicho sin incurrir en inconsistencias. En definitiva, que desde la perspectiva proporcionada por el realismo interno, de un modo similar a como acontecía con los planteamientos más consistentes de K. Popper y T. Kuhn, en el análisis y explicación del desarrollo científico y del concepto de verdad el mundo de los objetos independientes de la mente no puede jugar ningún papel. No es la naturaleza de lo en sí la que da su asentimiento o pone su veto a nuestro mundo nocional, sino el mundo conceptualizado de nuestra experiencia. 
Para el realismo interno la verdad de un enunciado no puede consistir en la correspondencia con los hechos, sino en el ajuste del enunciado en cuestión con el mundo conforme éste se presenta al observador u observadores. 0 , como se nos dice expresamente en Representation and Reality: "a statement is true of a situation just in case it would be correct to use the words of which the statement consists in that way in describing the situation" (Putnam, 1988, p. 115).

El concepto de verdad sigue siendo necesario en la explicación del desarrollo científico, pero esa noción de verdad no puede seguirse entendiendo por más tiempo en el sentido clásico de correspondencia entre enunciados por un lado y "lo que está realmente ahî" por el otro, sino entre enunciados y "lo que (parafraseando a T. Kuhn) la naturaleza realmente semeja". En una versión muy próxima al texto de Putnam que se acaba de citar, diremos de un enunciado singular cualquiera que es verdadero si resulta adecuado en la descripción de una situación o, lo que vendría a ser lo mismo, si existe una situación que acepta ser codificada por ese enunciado. Y, por extensión, diremos también de una teoría que es verdadera o que se aproxima a la verdad, si los enunciados básicos de los que ella puede dar cuenta, constituyen una descripción racionalmente aceptable de las experiencias para las que la teoría ha sido propuesta.

En el supuesto de que queramos proseguir hablando del concepto de verdad como correspondencia podemos hacerlo, pero a condición de que no pretendamos establecer esa correspondencia entre lo que los enunciados dicen y estados de cosas independientes de la mente o del discurso, sino entre lo que dicen los enunciados y aquello como lo que, bajo la perspectiva conceptual proporcionada por esos enunciados, la naturaleza es experimentada. La alternativa al realismo metafísico propuesta por Putnam no implica que todo sea lenguaje y nada más que lenguaje. Existen hechos y esos hechos son descubiertos y no legislados por nosotros, "pero esto es algo que sólo puede de- 
cirse cuando se ha adoptado un modo de hablar, un 'esquema conceptual' "(Putnam, 1988, p. 114).

Muchas veces la naturaleza nos dice "no", cuando nosotros esperábamos un "si". El sentido que le habíamos atribuido y que se recoge en nuestro lenguaje, no encaja con el que se constituye al nivel de los actos experimentales de observación. En 1769 Herschel había identificado a Urano como una estrella; cuando doce años después pudo observarla de nuevo con un telescopio más perfeccionado, descubre que el tamaño aparente del disco no era el usual para las estrellas, por lo que decide llevar a cabo un examen más minucioso. Ese examen le permite observar el movimiento de Urano entre las estrellas. Evidentemente no se trataba, pues, de una estrella; la naturaleza observada acababa de decir "no". De acuerdo con los esquemas conceptuales de Herschel, el planeta Urano no acepta ser codificado como una estrella, pero sí como un cometa, que es como de hecho lo identificó Herschel. Sólo unos meses más tarde, después de varios intentos infructuosos para ajustar el movimiento observado a una órbita de cometa, Lexell sugirí que probablemente se tratase de una 6rbita planetaria. Para poder observar a Urano como un planeta y no como una estrella o un cometa, se hace necesario disponer del marco conceptual proporcionado por la astronomía. Sin ese marco que habla de órbitas, tamaños aparentes, etc. etc., nada hay en la naturaleza que nos permita distinguir a una estrella de un cometa o un planeta. Si en 1781 había dejado de ser verdad para Herschel que Urano no era una estrella se debe única y exclusivamente a que no encajaba como tal en sus esquemas conceptuales.

Sin lugar a duda este punto de vista comporta una cierta relatividad conceptual, pero la "relatividad conceptual" nada tiene que ver con el "relativismo". La relatividad conceptual no implica que no haya verdades que no puedan ser descubiertas, ni que el término "verdad" sea tan sólo un nombre para aquello sobre lo que un grupo de gente se pone de acuerdo (Putnam, 1978, p. 17). 
Desde el marco proporcionado por el realismo interno y las tesis de Popper y Kuhn que aquí he presentado como más consistentes, la meta a la que se dirige el desarrollo científico no es la abstracta verdad "absoluta" y "objetiva" de Popper, sino la consecución concreta de la coherencia ideal de todas nuestras creencias entre sí y con nuestras experiencias, tal como estas experiencias son representadas en nuestro sistema de creencias.

\section{BIBLIOGRAFIA}

Bhaskar, R., 1975, A Realist Theory of Science, Leeds Books, Leeds. Brink, Chris, 1989, "Verosimilitude: Views and Reviews", History and Philosophy of Logic, no. 10, pp. 181-201.

Harré. R., 1985, Varieties of Realism, Oxford, Blackwell.

Kemeny, J.G. y P. Oppenheim, 1956, "On Reduction", The Philosophical Studies, no. 7, pp. 6-19.

Koethe, J., 1979, “Putnam's Argument Against Realism", The Philosophical Review, no. 88, pp. 92-99.

Kuhn, T., 1962, The Structure of Scientific Revolutions, University of Chicago Press, Chicago, (2a.ed. 1970). [Vers. cast., La estructura de las revoluciones científicas, FCE, México, 1975.]

—, 1969, "Posdata: 1969" a The Structure of Scientific Revolutions (2a. ed. ampliada, 1970), Chicago.

- 1970 , "Reflections on My Critics", en Lakatos, I. y A. Musgrave (eds.), Criticism and the Growth of Knowledge, Cambridge University Press., Cambridge. [Vers. cast., La crítica y el desarrollo del conocimiento, Grijalbo, Barcelona, 1975.]

Leplin, J. (ed.), 1984, Scientific Realism, University of Califormia Press, Berkeley.

Merrill, G. H., 1980, "The Model Theoretic Argument Against Realism", Philosophy of Science, no. 47, pp. 69-81.

Nagel, E., 1961, The Structure of Science, Harcourt Brace, Nueva York. [Vers. cast., La estructura de la ciencia, Paidós, Barcelona, 1968.]

Newton-Smith, W. H., 1981, T/ue Rationality of Science, Routledge 
and Kegan Paul, Boston. [Vers. cast., La racionalidad de la ciencia, Paidós, Barcelona, 1987.]

Niiniluoto, I., 1977, "On the Truthlikeness of Generalizations", en

R. Butts, y J. Hintikka, (eds.), Basic Problems in Methodology and

Linguistics, Reidel, Dordrecht, pp. 121-147.

—, 1980, "Scientific Progress", Synthèse, no. 45, pp. 427-462.

, 1982, "What Shall We Do with Verosimilitude?", Philosophy

of Science, no. 49, pp. 181-197.

$\longrightarrow$ 1987, Thruthlikeness, Reidel, Dordrecht.

Oddie, G., 1986, Likeness to truth, Reidel, Dordrecht.

Pearce, D. y V. Rantala, 1982, "Realism and Reference: Some

Comments on Putnam", Synthèse, no. 52, pp. 439-448.

$\longrightarrow, 1982$, "Realism and Formal Semantics", Synthèse, no. 53, pp. 39-53.

Popper, K., 1935, Logik der Forschung, Springer, Viena. [Vers. cast., La lógica de la investigación científica, Tecnos, Madrid, 1962.

$\longrightarrow, 1965$, Conjectures and Refutations. The Growth of Scientific Knowledge, 2a. ed., Basic Books, Nueva York. [Vers. cast., Conjeturas y refutaciones. El desarrollo del conocimiento científico, Paidós, Buenos Aires, 1967. (2a. ed. 1983).]

Putnam, H., 1975, Mind, Language and Reality. Philosophical Papers, vol. II, Cambridge University Press, Cambridge.

$\longrightarrow, 1978$, Meaning and the Moral Sciences, Routledge and Kegan Paul, Londres.

- , 1981, Reason, Thruth and History, Cambridge University Press, Cambridge. [Vers. cast., Razón, verdad e historia, Tecnos, Madrid, 1988.

- 1982, "Three Kinds of Scientific Realism", The Philosophical Quaterly, no. 32, pp. 195-200.

- 1983, Realism and Reason. Philosophical Papers, vol. III, Cambridge University Press, Cambridge.

—, 1987, The Many Faces of Realism, Open Court, La Salle (IIl.). , 1988, Representation and Reality, the MIT Press, Cambridge. , 1989, "Models Theory and the 'Faculty' of Semantics" en A. George (ed.), Reflections on Chomsky, Basil Blackwell, Cambridge.

Quintanilla, M. A., 1982, “La verosimilitud de las teorías", en I Congreso de teoría y metodología de las ciencias, vol. I, Oviedo, pp. $473-489$. 
Rivadulla, A., 1984, Filosofia actual de la ciencia, Madrid, Ed. Nacional.

Sanmartín, J., 1984, "A vueltas con el concepto de verosimilitud", Madrid.

Tuomela, R., 1979, “Putnam's Realism”, Theoria, no. 45, pp. 114126.

, 1979, Scientific Change and Approximation, North Holland, Amsterdam.

_-, 1985, Science, Action, and Reality, Reidel, Dordrecht.

Recibido: 11 de marzo de 1991 . 
The aim of this paper is to make a plea for a new philosophical approach, or a new meta-theoretic account alternative to the ones represented by realism and instrumentalism, in the Philosophy of Science, and that will enable us to be more successful both in dealing with a rational explanation of the process of scientific development as well as with the notion of truth that is implied by the former. This new approach is consistent with the epistemology of Logik der Forschung, goes along with T. Kuhn's contribution, and gets its most explicit formulation in the "internal realism" of the last Putnam.

The argument takes the following steps: 1) I begin with a very brief mention of the neopositivist approach to scientific development, concluding at his point that if anything has survived in that account of scientific development it can only be the idea of "convergence", linked to the notion of "reduction". 2) The notion of convergence in K. Popper's work is then analysed, showing that this notion can be understood in two quite different senses, depending on whether what we mean is Popper in its Logik der Forschung or the tarskian Popper, i.e., the Popper of verisimilitude. 3) I show then that the only notion of convergence that necessarily follows from the Logik der Forschung is consistent with T. Kuhn's approach and will only be slightly qualified by him, in such a way that Kuhn's qualified notion of convergence fits perfectly well within the framework of a falsationist epistemology. The strong controversy historically held between Popper's and Kuhn's followers that originated from The Structure of Scientific Revolutions by Kuhn, can only be understood when we mean by Popper the tarskian Popper, and hold a misleading interpretation of Kuhn's work. 4) A new meta-theoric approach is reached both in connection with the analysis and explanation of scientific progress and with the not less troublesome concept of truth. This new approach is very akin to the "internal realism" held by Putnam from 1976 onwards. Within the framework of this new approach an epistemic notion of truth is contended for, while the goal of scientific development is now not the search for an "absolute", "objetive" truth, but the concrete achievement of the ideal coherence of the whole of our beliefs between themselves, the coherence of the whole of our beliefs with the whole of our experiences, taking our experiences in the way they are represented in our system of beliefs. 